\title{
Gamma-ray Irradiation Impairs Dendritic Cell Migration to CCL19 by Down-regulation of CCR7 and Induction of Cell Apoptosis
}

\author{
Cong Liu*, Jin Lin*, Luqian Zhao*, Yanyong Yang, Fu Gao, Bailong Li, Jianguo Cui ${ }^{凶}$, Jianming Cai ${ }^{凶}$ \\ Department of Radiation Medicine, Faculty of Naval Medicine, Second Military Medical University, Shanghai, PR China. \\ * These authors contributed equally to this work.
}

Corresponding author: Jianming Cai and Jianguo Cui. Address: Department of Radiation Medicine, Second Military Medical University; 800, Xiangyin Road 200433, Shanghai; P.R. China Fax: +86-21-81871148. E-mail: caijianming882003@163.com and mustardcc@yahoo.com.cn.

Received: 2010.09.30; Accepted: 2011.02.05; Published: 2011.02.14

\begin{abstract}
Dendritic cells (DCs) are the most potent antigen-presenting cells and play a crucial role in the regulation of immune response and migration of DCs into secondary lymphoid tissues also play an important role in the initiation of innate and adaptive immunity. Radiation therapy is now a routine treatment for certain types of cancer and over 20 percent of cancer patients will require radiation therapy during the treatment of their disease. However, the influence of ionizing irradiation on the migratory ability of DCs is largely unknown. In this article, we report that $\gamma$ ray irradiation can significantly inhibit LPS-triggered up regulation of CCR7 expression and PGE2 production by DC, thus impairing DC migration towards CCLI9 in vitro and in vivo. Moreover, $\gamma$ ray exposed DC also displayed an increased apoptosis rate and decreased cell viability. Furthermore, we demonstrate that exogenous PGE2 can partly reduce the gamma-ray induced migratory impairment and restored CCR7 expression of DC. Our work suggests that $\gamma$ irradiation affects DC function at multiple steps during the immune response including DC migration, and that PGE2, via control of CCR7 expression, is an important regulator of DC migration.
\end{abstract}

Key words: $\gamma$ ray Irradiation, Dendritic cell; Migration; CCR7; Apoptosis.

\section{Introduction}

Dendritic cells (DCs, DC) are the most potent antigen-presenting cells (APC) and play a critical role in immune regulation, ranging from tolerance induction and the prevention of autoimmunity, to the induction of anti-tumor immunity and the protection against infectious agents [1-4]. Genetic tagging shows DCs are specialized and regulated antigen processing machines and they play critical role in epidermal viral immunity [5-7]. In-activation of host DCs can also prevent graft versus host disease in animal model [8, 9]. From ex vivo loading to in vivo targeting of DCs have developed many pharmacological targets of immunosuppressive drugs in immunotherapy [10, $11]$.
DCs originate from bone marrow precursors and migrate through the blood stream to almost every tissue, where they reside in an immature state. Studies also show that Langerhans cells can renew in the skin throughout life under steady-state condition [12]. At the site of an infection, DCs recognize and respond to common pathogen-associated molecular patterns (PAMP), including lipopeptides, lipopolysaccharides (LPS) and nucleic acids via Toll-like receptors (TLR) [13-15]. LPS activates DC and induces production of pro-inflammatory cytokines and other mediators via TLR4, thus triggering a complex phenotypic and functional maturation program of DC including up-regulation of the chemokine receptor CCR7 [16]. 
The CCR7 upregulation enables an increased responsiveness of DC to CCR7 ligands, which promotes DC migration from peripheral tissues to secondary lymphoid organs such as lymph nodes (LN) and the spleen where DC present antigens to T cells and initiate immune response [17]. The expression of CCR7 on mature DC is essential for their migration to the T-cell area of draining $\mathrm{LN}$ because this migration is guided by two ligands for CCR7, CCL19 (EBI1-ligand chemokine [ELC], macrophage inflammatory protein [MIP]-3 $\beta$ ), and CCL21 (secondary lymphoid-tissue chemokine [SLC], 6-Ckine), Both chemokines are expressed by stromal cells in the T-cell area of secondary lymphoid organs [18]. The essential role of CCR7 and its ligands in mature DC migration to $\mathrm{LN}$ was demonstrated in CCR7 deficient mice and plt/plt mice, which lack the ligands for CCR7 [17]. Because the migration of DC into secondary lymphoid organs is required for the initiation of specific immune responses, inhibition of DC migration into secondary lymphoid organs by inhibiting CCR7 expression is an efficient way to induce immunosuppression and tolerance. PGE2, derived from the metabolism of free arachidonic acid, exerts an important immunomodulatory role in DC differentiation and function [19]. Previous studies have shown that PGE2 is required for CCR7-expressed DC migration to its ligands, CCL19 and CCL21, and the importance of PGE2 for DC migration in vivo has also been shown in Ptger4-/- mice lacking the PGE2 receptor EP4 [20, 21]. These mice display impaired migration of Langerhans cells and reduced skin immune responses.

With the development of clinical radiation therapy and the nuclear industry, the ionizing radiation (IR) hazards to human health have attracted more and more attention [22, 23]. It is generally accepted that ionizing radiation is capable of causing cellular damage, immune-suppression and even cell death of many cell types, including immune cells [23]. The effect of IR on T and B cells has been extensively studied; however the influence of ionizing irradiation on DC function is largely unknown. In particular, the role of $\gamma$-irradiation on the migratory abilities of DC, is still not clear. Therefore, a study of radiation effects on DC migration would be very important.

In this study, we demonstrate that $\gamma$ - irradiation can significantly inhibit LPS-triggered upregulation of CCR7 expression and PGE2 production by DC, thus impairing DC migration towards CCR7 ligand CCL19 in vitro and in vivo. Moreover, $\gamma$ ray exposed DC also display an increased apoptosis rate and reduced cell viability which also promotes the migration impairment induced by IR. Most importantly, we demonstrate that exogenous PGE2 partly reduces the gam- ma-ray induced migratory impairment and restores the CCR7 expression of DC, but don't affect the apoptosis level of DC. Our work suggests that $\gamma$ - irradiation affects DC function at multiple steps during the immune response, including DC migration, and that PGE2, via control of CCR7 expression, is an important regulator of DC migration.

\section{Materials and Methods}

\section{I Mice and reagents}

Male wild-type C57BL/ 6 mice, 5-6 weeks of age, were purchased from Chinese academy of science (Shanghai, China). All mice were housed in a pathogen-free facility for all experiments. All animal experiments were undertaken in accordance with the National Institute of Health Guide for the Care and Use of Laboratory Animals, with the approval of the Laboratory Animal Center of Second Military Medical University, Shanghai. The approval ID for this study was 20090101. All efforts were made to minimize the number of animals used as well as their suffering. Recombinant mouse GM-CSF, IL-4 and CCL19 were purchased from Peprotech Systems (rocky Hill, NJ, Minneapolis, MN). PE-labeled CCR7 Ab was purchased from ebioscience (San Diego, CA). ELISA kits for murine PGE2 and IL-10 were purchased from R\&D Systems (Minneapolis, MN). Lipopolysaccharide (LPS) were from Sigma Company (USA). Cell viability kits (CCK8) and CFSE were from Dojindo Laboratories (Kumamoto, Japan) and Annexin V-FITC and propidium iodide Apoptosis Detection Kits were from Bipec Biopharma, (Massachusetts, USA.)

\subsection{Generation of mouse bone marrow (BM)-derived DC}

Mouse immature DC was prepared from bone marrow progenitors according to published method of our university with minor modifications [17, 24, 25]. Bone marrow mononuclear cells were prepared from murine (5-6 weeks old) femur bone marrow suspensions by depletion of red cells, and then cultured at a density of $2 \times 10^{6}$ cells $/ \mathrm{ml}$ in 6-well plates in RPMI1640 medium supplemented with 10\% FCS, $10 \mathrm{ng} / \mathrm{ml}$ recombinant murine GM-CSF and $1 \mathrm{ng} / \mathrm{ml}$ recombinant murine IL-4 (peprotech Systems). Non-adherent cells were gently washed out on the third day of culture; the remaining loosely adherent clusters were used on day 5 as immature DC. On day 5, cells were exposed to gamma-rays and treated with LPS (1ug/ml, 24hours) according to a published method [20, 21]. 


\subsection{Gamma Irradiation}

A ${ }^{60} \mathrm{Co}$ theratron $(\mathrm{Fyc}-50 \mathrm{H})$ machine was used to deliver the radiation (Irradiation Center, Faculty of Naval Medicine, Second Military Medical University, China) DCs were exposed to different doses of radiation, (Dose rate: $1 \mathrm{~Gy} / \mathrm{min}$ ) depending upon the requirement of the study according to our published method [26-28].

\subsection{In vitro $D C$ chemoattraction assay}

To evaluate the responsiveness of $\gamma$ ray exposed DC to CCL19 (macrophage inflammatory protein-3ß), an in vitro chemoattraction assay was performed in 24-well transwell chambers with $5 \mu \mathrm{m}$-pore size polycarbonate filters (Corning Costar, Cambridge, MA). $100 \mathrm{ng} / \mathrm{ml}$ CCL19 diluted in RPMI 1640 containing $0.5 \%$ bovine serum albumin (BSA) were added to lower wells in a volume of $0.6 \mathrm{~mL}$; $0.1 \mathrm{~mL}$ RPMI 1640 containing $2 \times 10^{5} \mathrm{DC}$ was added to the upper wells and chambers incubated for 4 hours at $37^{\circ} \mathrm{C}$. Directed migration was expressed as the number of cells that had migrated to the lower chamber, as counted by FACS as described previously [20,21].

\subsection{In vivo DC migration assays}

DC were labeled with $5 \mu \mathrm{M}$ carboxyfluorescein diacetate succinimidyl ester (CFSE; Dojindo, Japan) in PBS at $37^{\circ} \mathrm{C}$ for 30 minutes and washed twice immediately before i.v. injection into C57BL/ 6 mice. 24 hours later, the skin of injected cite and the draining inguinal LN were harvested and processed as described previously $[17,24,25]$. Single cell suspensions were prepared, stained with CD11c-PE (eBioscience) and analyzed by flow cytometry.

\subsection{Assay for CCR7 expression}

Prior to fluorescent antibody staining, all cells were incubated with CD16/CD32 antibody at a concentration of $1 \mu \mathrm{g} / 10^{6} \mathrm{cells} / 100 \mu \mathrm{l}$ for 15 minutes at $4^{\circ} \mathrm{C}$ to block Fc receptors. Fluorescent antibodies (CCR7-PE, ebioscience) and respective isotype controls were then added at a concentration of $1 \mu \mathrm{g} / 10^{6} \mathrm{cells} / 100 \mu \mathrm{l}$, and cells incubated for a further 30 minutes at $4^{\circ} \mathrm{C}$. The cells were washed once with ice-cold PBS (pH 7.2) containing $0.1 \% \mathrm{NaN}_{3}$ and $0.5 \%$ BSA, and resuspended in $300 \mathrm{ml}$ PBS. Flow cytometry was performed using a FACS Calibur and data analyzed using CellQuest software (both from Becton Dickinson, Mountain View, CA) according to a published method [20, 25].

\subsection{ELISA assay}

IL-10 and PGE2 contained in the culture supernatants of DC with or without $\gamma$ ray exposure and before or without LPS stimulation were measured by
ELISA (R\&D, Minneapolis, MN) according to the manufacturer's instructions and some published method [25].

\subsection{Apoptosis assay.}

After different treatments, DCs were labeled with annexin V-FITC and propidium iodide (PI) provided by BIPEC, following manufacturer's instructions. Samples were examined by fluorescence-activated cell sorter (FACS) analysis, and the results were analyzed using Cell-Quest software (Becton Dickinson, San Jose, CA) [25].

\subsection{Cell Viability Analyses}

Trypan blue exclusion test were used for detecting cell viability as previously described. Briefly, after different treatments, DCs were labeled with Trypan Blue. The cellular morphology was observed using Olympus BX60 fluorescent microscope equipped with Retiga 2000R digital camera. Average percentage of Trypan Blue positives and negative cells were calculated in 5-7 randomly selected high power field (HPF). Trypan Blue negative cells were regarded as living cells. Alternatively, cell viability was determined by MTT assay and CCK8 assay (cell counting kit 8, Dojindo Laboratories,Kumamoto, Japan). Measuring the optical density (OD) at $450 \mathrm{~nm}$ with microtiter plate reader, we calculated cell viability by standard curve.

\subsection{Statistical analysis}

Comparisons between experimental groups and relevant controls were performed by Student's t-test. $\mathrm{P}<0.05$ was considered a statistically significant difference.

\section{Results}

\section{I $\gamma$-ray exposure inhibits CCLI 9 induced DC migration in a dose dependent way}

One characteristic of mature DC is their ability to migrate to secondary lymphoid organs where DC present antigens to $\mathrm{T}$ cells and initiate an immune response [20, 21]. Therefore, inhibition of DC migration to secondary lymphoid organs is an efficient way to induce immunosuppression and tolerance. To confirm whether ionizing radiation affects DC migration, we examined in vitro migration of DC toward CCL19, the chemokine chemotactic for mature DC. As can be seen from figure 1, LPS-matured DC responded to CCL19 much more efficiently than immature DCs (0 Gy, $\mathrm{P}<0.05$; Figure 1A). In contrast, after pre-exposure to $\gamma$ irradiation, LPS-matured DC responded less well to CCL19 (Figure 1A). Interestingly, $\gamma$-irradiation inhibited LPS -induced DC migration to CCL19 in a 
dose dependent manner. In a time course study system, DC were pre-exposed to 2 Gy of $\gamma$ rays irradiation or left unexposed, and then $0,2,6,12,24,48$ and 72 hours later, the migration capacity of these DC was detected by FACS analysis. Time course study showed that $\gamma$-irradiation inhibited DC migration to CCL19 in a time related manner (Figure 1B and 1C). Thus, $\gamma$-ray exposure inhibits CCL19 induced DC migration in a dose and time related way.
A

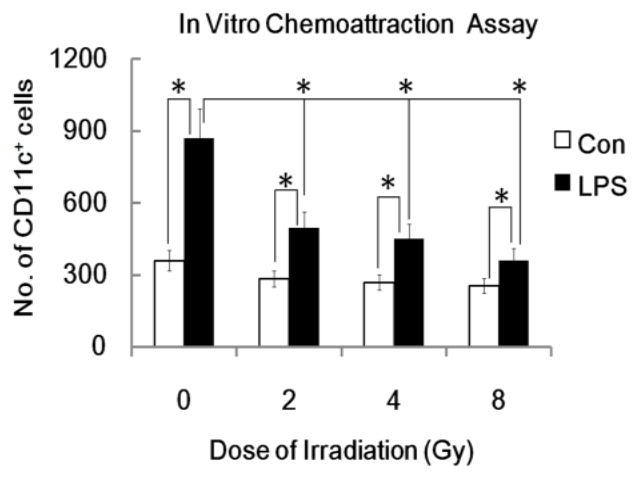

C

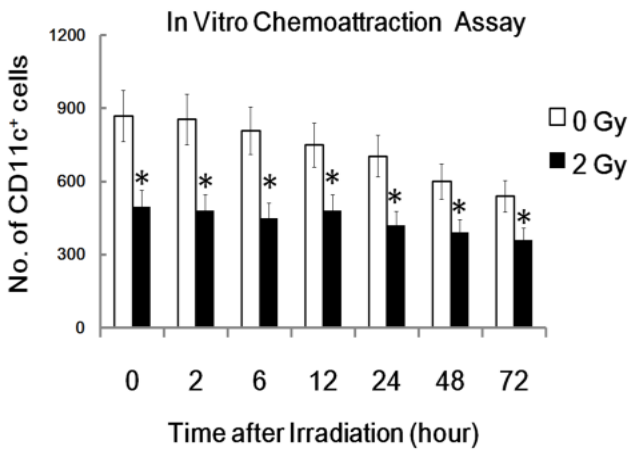

B In Vitro Chemoattraction Assay

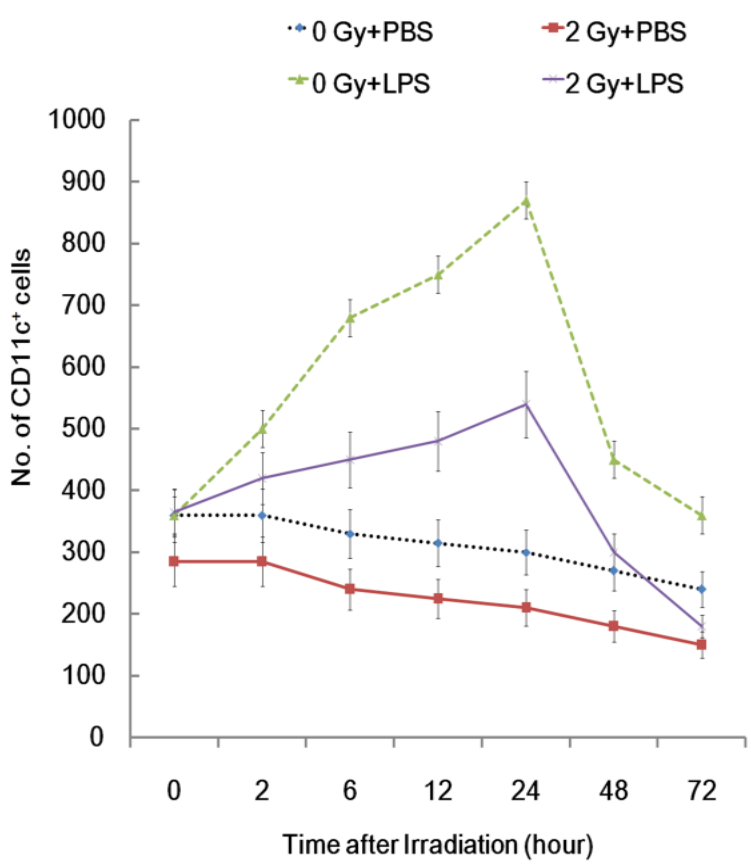

Figure I. Effect of $\gamma$ radiation exposure on DC migration. DC with different treatment were subjected to an in vitro chemoattraction assay to evaluated their migration capacity to CCLI9 as described detailed in Materials and Methods section and in many other previously studies [20,2I]. Each experiment was performed in triplicate and repeated at least three times. Data is expressed as mean number of migrated DC cells; \pm S.D. (A): DC were treated with or without $\gamma$ ray irradiation (Dose 0, 2, 4, 8Gy as indicated, Dose rate: IGy/min) with or without LPS (lug/ml) for 24 hours. Then these DC were subjected to in vitro chemoattraction assay to detect their migration capacity. (B): DC were treated with or without 2 Gy $\gamma$ ray irradiation (Dose rate: IGy/min) with or without LPS (Iug/ml) for the indicated time $(0,2,6,12,24,48$ and 72 hours). Then these DC were subjected to in vitro chemoattraction assay to detect their migration capacity. (C) : DC were treated with or without $2 \mathrm{~Gy} \gamma$ ray irradiation (Dose rate: IGy/min) and then after further incubation for the indicated time $(0,2,6,12,24,48$ and 72 hours), these DC were treated with LPS ( l ug/ml) for another 24 hours and then they were subjected to in vitro chemoattraction assay to detect their migration capacity by FACS assays. *: $P<0.05$.

\section{2 $\gamma$-irradiation inhibits LPS-induced CCR7 ex- pression and PGE2, ILI 0 secretion by DC}

It's well known that LPS induces maturation of DC with up-regulation of CCR7 which binds to the ligand CCL19 and directs DC migration to secondary lymph organs, a process critical for DC to initiate an immune response. PGE2, derived from the metabo- lism of free arachidonic acid, exerts an important immunomodulatory role in DC differentiation and function [17]. Prostaglandin E2 may be the key factor for CCR7 surface expression and migration of monocyte-derived dendritic cells [20]. Additionally, IL-10 may also down-regulate CCR7 expression by myeloid dendritic cells. To confirm that the reduced migration of DC by $\gamma$-irradiation is associated with the inhibited 
CCR7 expression and altered PGE2 and IL10 production, we investigated FACS assay to study CCR7 expression and ELISA assay for PGE2 and IL10 in $\gamma$ ray exposed DC with or without LPS stimulation. DC were pre-exposed to $\gamma$ rays or left unexposed, and then stimulated with LPS $(1 \mathrm{ug} / \mathrm{ml})$ for $24 \mathrm{~h}$ for FACS analysis of the cell and ELISA assay of the supernatant. Gamma-irradiation significantly inhibited LPS-induced CCR7 expression in DC (Figure $2 \mathrm{~A}$ and B). Similarly, in a time course study system, 2Gy Gamma-irradiation also significantly inhibited CCR7 expression in DC in a time related manner (Figure 2 C). These data indicate the impaired LPS-induced up-regulation of CCR7 expression in $\gamma$ ray-treated DC may account for their impaired migratory ability. LPS-induced PGE2 and IL10 production by DC were also been found to be significantly inhibited by $\gamma$ ray in a dose and time related manner (Figure $3 \mathrm{~A}$ and B). This suggests that $\gamma$ ray irradiation can significantly inhibit LPS triggered up-regulation of CCR7 expression by down regulation of PGE2 production by DC, thus impairing DC migration towards CCR7 ligand CCL19 in vitro, while IL10 may not participate in the IR induced migration impairment of DC.
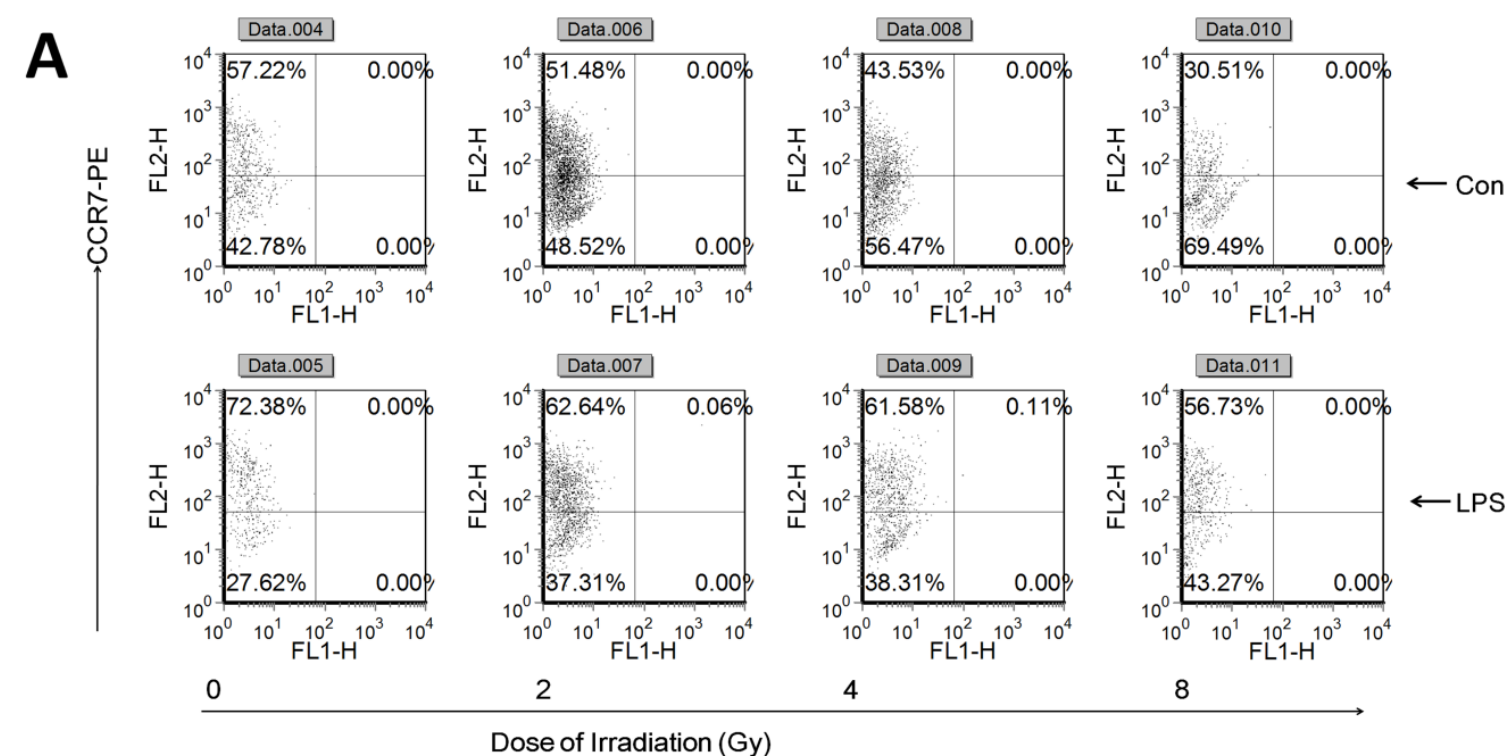

0

$$
2
$$

\section{4} 8

B

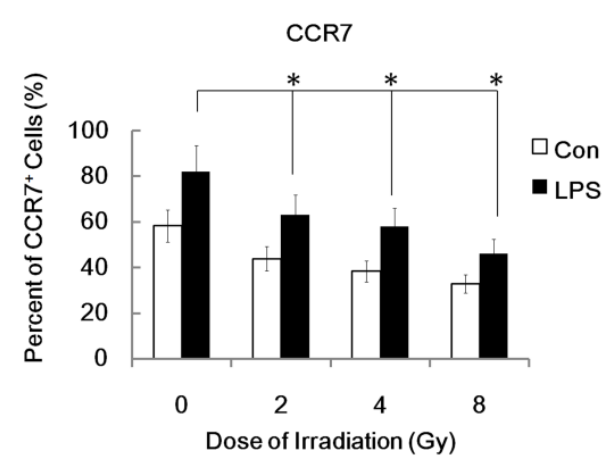

C

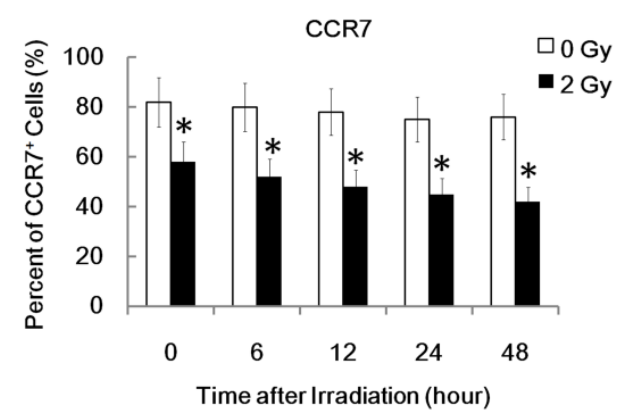

Figure 2. Gamma-radiation down-regulated CCR7 expression by DC in a time and dose related manner. DC with different treatment was labeled with CCR7-PE antibody for detection of the CCR7 positive cell percent by FACS assays. Each experiment was performed in triplicate and repeated at least three times. Data is expressed the mean CCR7 positive cell rate \pm S.D. *: $P<0.05$; NS: No Significant difference detected. (A/B) : DC were treated with or without $\gamma$ ray irradiation (Dose 0, 2, 4, 8Gy as indicated, Dose rate: I Gy/min) with or without LPS (I ug/ml) for 24 hours and then the CCR7 positive cell rate in these DC were detected by FACS assay. A showed a representative data of at least three independent experiments. B showed the statistical data. (C) : DC were treated with or without $2 \mathrm{~Gy} \gamma$ ray irradiation (Dose rate: IGy/min) and then after further incubation for the indicated time $(0,2,6,12,24,48$ and 72 hours), these DC were treated with LPS ( l ug/ml) for another 24 hours and then the CCR7 positive cell rate in these DC were detected by FACS assay. *: $P<0.05$. 

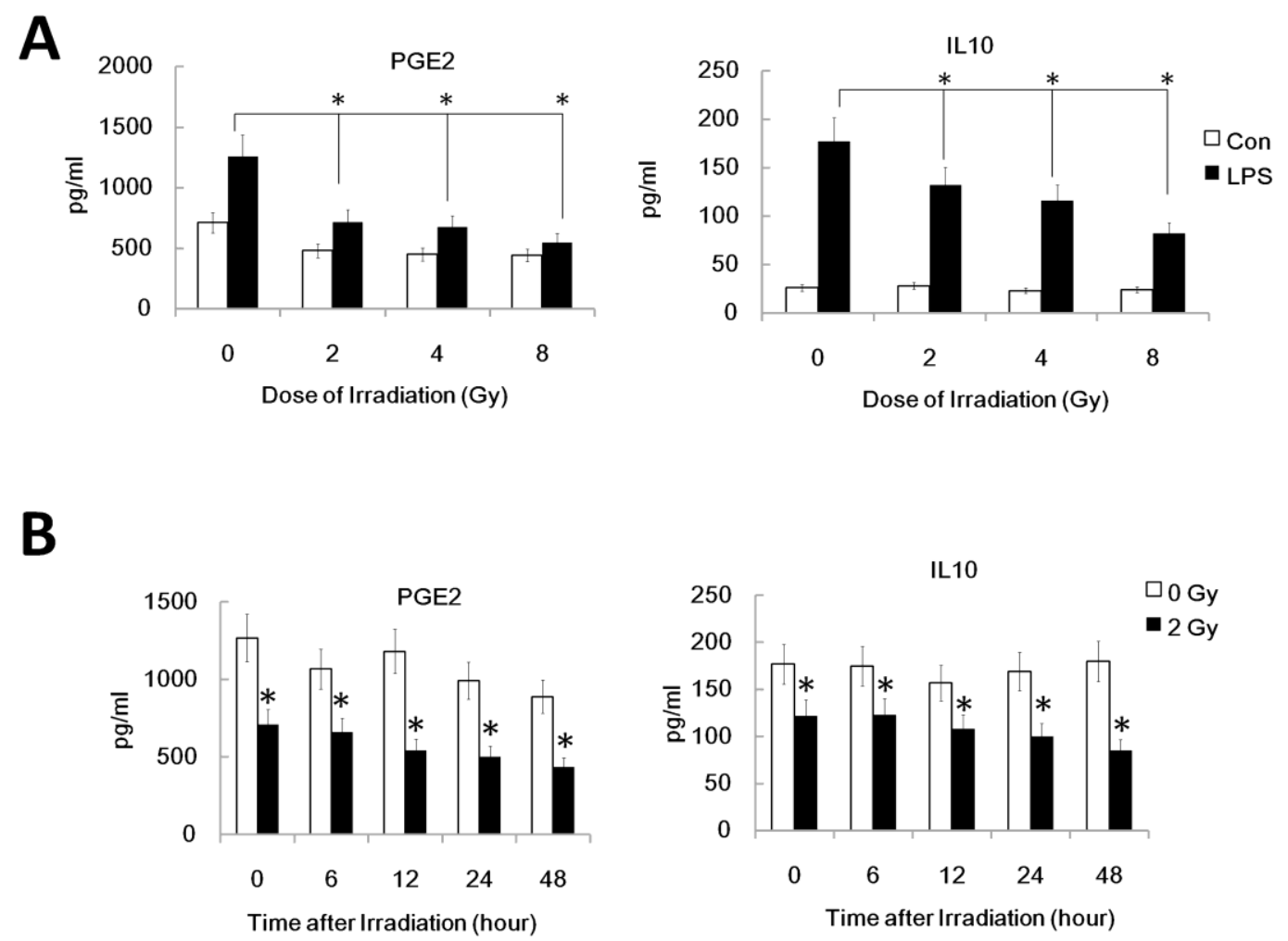

Figure 3. Gamma-irradiation down-regulated PGE2 and ILIO secretion by DC. The supernatant of DC with different treatment was collected and subjected to ELISA assay for detection of the PGE2 and ILIO level. (A): DC were treated with or without $\gamma$ ray irradiation (Dose 0, 2, 4, 8Gy as indicated, Dose rate: IGy/min) with or without LPS (I ug/ml) for 24 hours and then the PGE2 and ILIO level in the culture supernatant were detected by ELISA assay. (B): DC were treated with or without 2 Gy $\gamma$ ray irradiation (Dose rate: I Gy/min) and then after further incubation for the indicated time $(0,2,6,12,24,48$ and 72 hours), these DC were treated with LPS (lug/ml) for another 24 hours and the PGE2 and ILI 0 level in the culture supernatant were detected by ELISA assay. *: $\mathrm{P}<0.05$.

\subsection{Exogenous PGE2 partly rescues the $\gamma$ ray-induced migratory impairment of DC and restores CCR7 expression.}

To further investigate the role of the reduced PGE2 in $\gamma$ ray mediated inhibition of DC migration, we added exogenous PGE2 into the DC migration assay, and found the addition of exogenous PGE2 $(1 \mathrm{uM})$ partially restored the migratory capacity of $\gamma$ ray -treated DC in chemo-attraction assays (Figure $4 \mathrm{~A})$. We also found that exogenous PGE2 partially restored CCR7 expression in $\gamma$ ray treated and LPS-stimulated DC (Figure 4B). These results are consistent with reports [20] showing that PGE2 regulates the expression of CCR7 in DC and influences their migration. However, we also found that PGE2 restores CCR7 expression much more effectively than it rescues of migratory impairment of DC induced by IR; PGE2 also acts more effectively on DC irradiated at $2 \mathrm{~Gy}$ than $8 \mathrm{~Gy}$ (Figure $4 \mathrm{C}$ ). These results suggest that the inhibition of DC migration by $\gamma$ ray may be partially due to its inhibition of PGE2 production, which in turn results in a defective CCR7 expression in DC, thus leading to the impaired migration of DC to CCL19. However, there must be other mechanisms also involved in $\gamma$ ray induced DC migration impairment.

\section{$3.4 \gamma$ ray induced apoptosis also participates in DC migration impairment.}

To confirm whether ionizing radiation affects the DC apoptosis rate, we examined in annexinV-FITC and propidium iodide (PI) double staining assay to determine the apoptosis rate of DC. We also used cell count assays to inspect DC cell viability. As expected, IR can induce DC apoptosis in a dose dependent manner (Figure $5 \mathrm{~A}$ and B). Gamma irradiation can also inhibit DC cell viability in a dose dependent manner (Figure 5C). These data suggest that $\gamma$ radiation can induce DC apoptosis and inhibit DC viability in a dose dependent manner.

To confirm that gamma ray induced apoptosis also participates in DC migration impairment, we 
next used FACS assay to value the apoptosis rate of migrated DC and not-migrated DC after different dose of irradiation (migrated DC means the DC that have migrated/transwelled in a transwell assay; not-migrated DC means the DC that have not migrated/transwelled in a transwell assay). We found that migrated DC show a much lower apoptosis rate than not-migrated DC in every group (Figure 6A). Indeed, the apoptosis rate of migrated DC was very low, even after 8Gy IR treatment. Finally, we used another positive control of apoptosis DC to confirm our theory that apoptosis can inhibit DC migration. We used activation induced apoptosis model as our positive control of DC and we found that DC treated with LPS for about $72 \mathrm{~h}$ to $96 \mathrm{~h}$ result in high apoptosis rate (Figure 6B), and these cells also show less ability to migrate to CCL19 than normal DC treated with LPS for $18 \mathrm{~h}$ to $24 \mathrm{~h}$, although they have similar CCR7 expression (Figure 6B). However, although PGE2 can partly rescues the $\gamma$ ray-induced migratory impairment of DC and it restores CCR7 expression, exogenous PGE2 have no effect on the apoptosis rate induced by $\gamma$ ray (Figure $6 \mathrm{C}$ ). Thus, $\gamma$ ray induced apoptosis also participates in DC migration impairment.

\section{A}

\section{In Vitro Chemoattraction Assay}

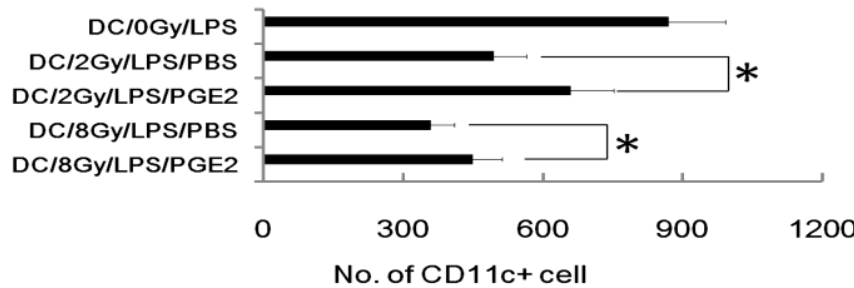

B

No. of CD11c+ cell
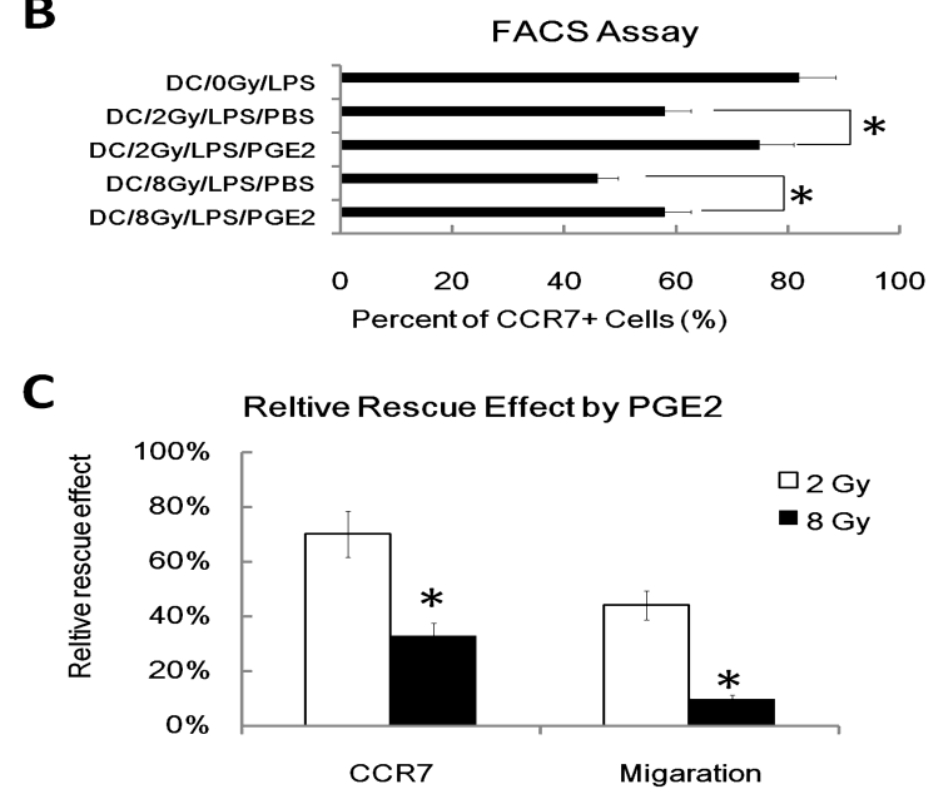

Figure 4. Exogenous PGE2 reduces the $\gamma$ radiation-induced migratory impairment of DC and restores CCR7 expression. DC were treated with or without $\gamma$ ray irradiation (Dose 0,2 , and $8 \mathrm{~Gy}$ as indicated, Dose rate: IGy/min) with LPS ( l ug $/ \mathrm{ml}$ ) or LPS (l ug/ml) and PGE2 (luM) together for 24 hours, then these DC were subjected to an in vitro chemoattraction assay to evaluated their migration capacity (A) as described in figure I and to be labeled with CCR7-PE antibody for detection of the CCR7 positive cell percent by FACS assay (B) as described in figure 2. Relative capacity of PGE2 restoration was also calculated (C). (A): Exogenous PGE2 reduces migration inhibition of $\gamma$ radiation treated DC to CCLI9. DC was exposed to indicated dose of $\gamma$ radiation, stimulated with LPS ( $\mathrm{lug} / \mathrm{ml}$ ) or LPS (l ug/ml) and PGE2 (luM) together for 24 hours, and then evaluated for responsiveness to CCLI 9 by the in vitro chemoattraction assay. (B): Exogenous PGE2 partially restores CCR7 expression. DC was exposed to indicated dose of $\gamma$ radiation, stimulated with LPS (lug/ml) or LPS (lug/ml) and PGE2 (I UM) together for 24 hours, and then be labeled with CCR7-PE antibody for detection of the CCR7 positive cell percent by FACS assay. (C): Relative capacity of PGE2 restoration was calculated by the ratio of relative inhibition capacity on DC by radiation with Exogenous PGE2 VS. Relative inhibition capacity on DC by radiation without Exogenous PGE2. *: $\mathrm{P}<0.05$. 

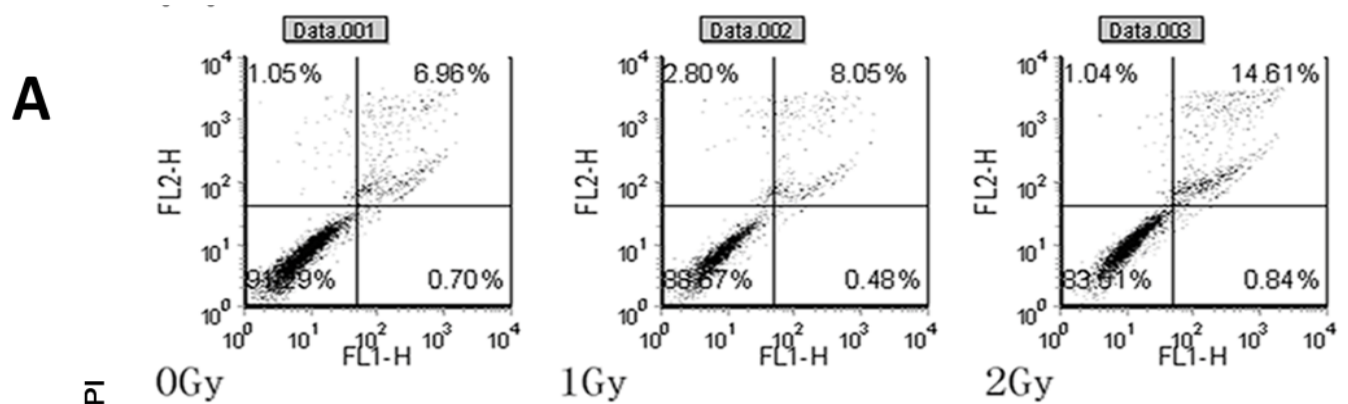

ฉ $\quad$ G $y$

$1 \mathrm{~Gy}$

$2 \mathrm{~Gy}$
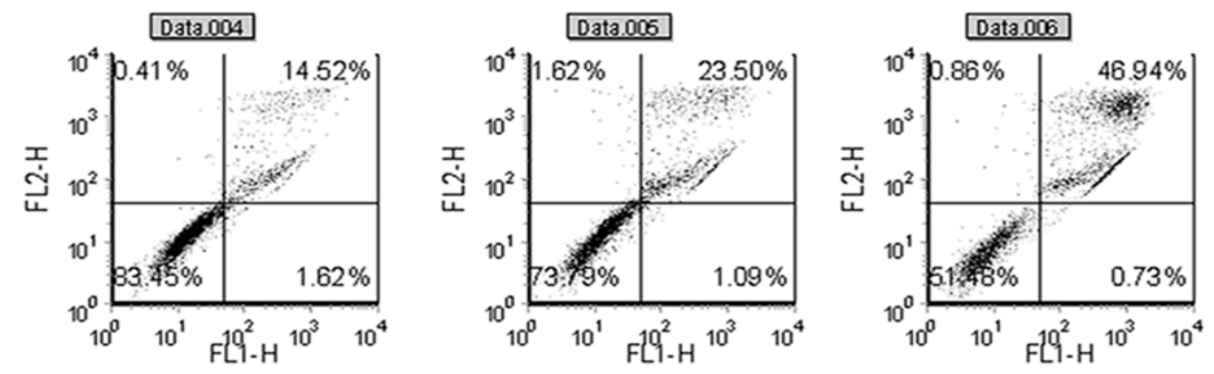

4Gy

8Gy

$16 \mathrm{~Gy}$
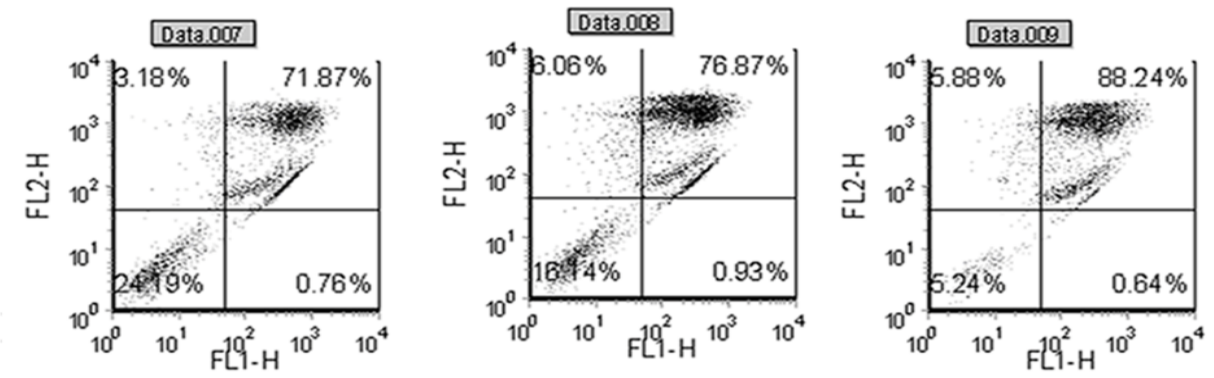

32Gy

48Gy

64Gy

AnnV-FITC

B
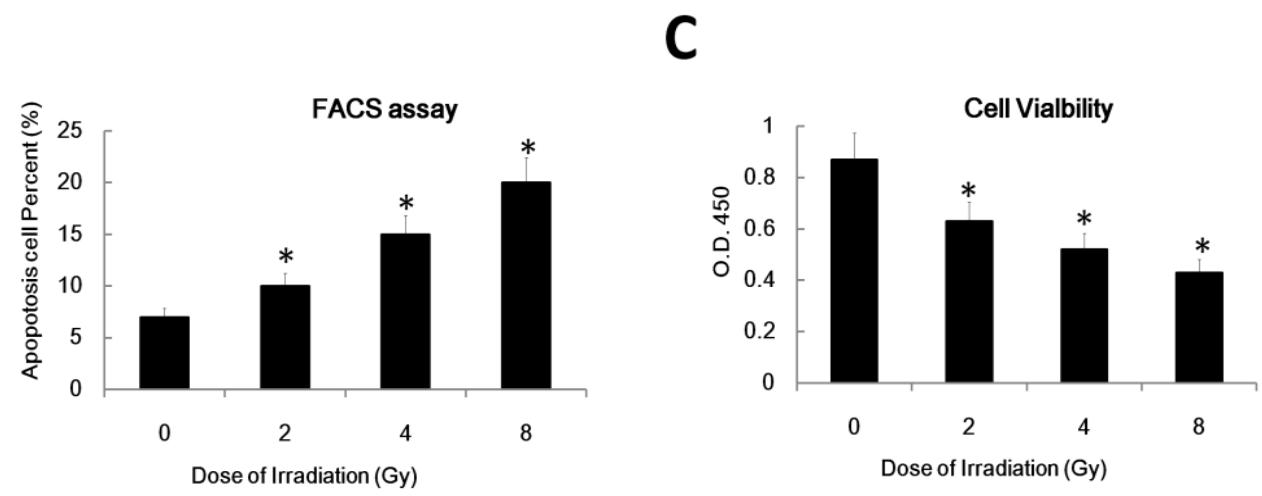

Figure 5. $\gamma$ radiation induced $D C$ apoptosis in a dose dependent manner. $(A / B)$ : $D C$ were treated with different doses( as indicated, dose rate: IGy/min) of $\gamma$ radiation and then 24 hours later was labeled with annexinV-FITC and PI for detecting of apoptosis rate by FACS assay. A showed a representative data of at least three independent experiments. B showed the statistical data. (C): DC was treated with different doses of $\gamma$ radiation and then 24 hour later the cell viability of these DC was detected by the CCK8 or MTT assay. Data is expressed as mean absorbance ratio at 450nM \pm S.D. *: P $<0.05$. 
A

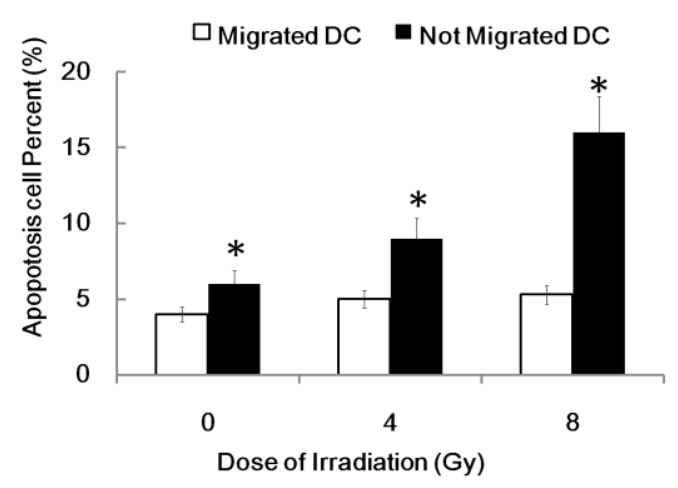

B

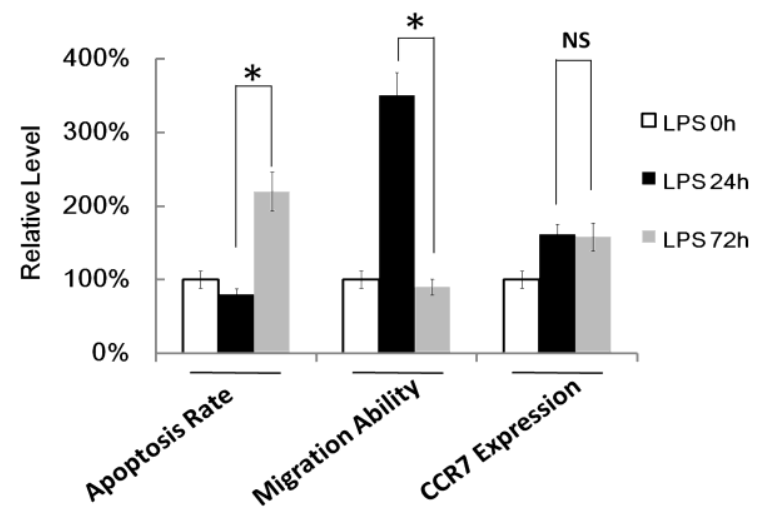

C

PGE2 on the Apoptosis Rate of DC

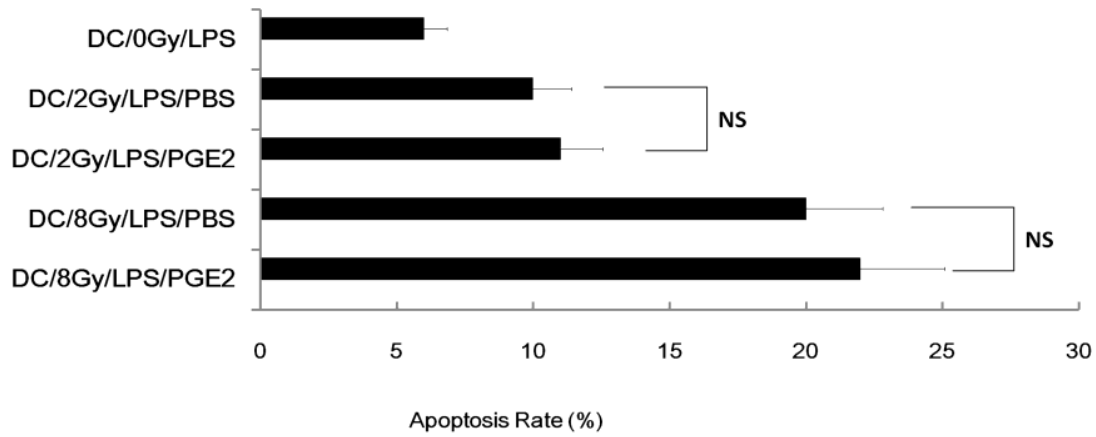

Figure 6. $\gamma$ ray induced apoptosis also participates in DC migration impairment. (A): DC were treated with or without $\gamma$ ray irradiation (Dose 0, 4 and $8 \mathrm{~Gy}$ as indicated, Dose rate: IGy/min) with LPS (I ug/ml) for 24 hours, then these DC were subjected to an in vitro chemoattraction assay. The migrated DC and the DC remained un-migrated were both collected and subjected to Apoptosis assay by FACS. Migrated DC and not migrated DC were referring to the result in the in vitro chemoattraction assay. (B): Apoptosis rate, migratory ability and CCR7 expression level of DC treated with LPS (I ug/ml) for 0,24 or 72 hours. Data showed the relative level compared with DC without LPS (LPS Ohour). (C): DC were treated with or without $\gamma$ ray irradiation (Dose 0,2 and $8 \mathrm{~Gy}$ as indicated, Dose rate: IGy/min) with LPS (lug/ml) or LPS (lug/ml) and PGE2 (I UM) together for 24 hours, then these DC was labeled with annexinV-FITC and PI for detecting of apoptosis rate by FACS assay. *: P < 0.05; NS: No Significant difference detected.

\section{$3.5 \gamma$-ray exposure inhibits DC migration in vivo}

To trace the in vivo migration of the IR-modified DC, $1 \times 10^{6}$ CFSE labeled irradiated or un-exposed control DCs were injected i.v. into C57BL/6 mice. Twenty four hours post-injection, the skin of injected cite and the draining inguinal LNs (lymph node) were dissected and analyzed for the presence of labeled DC by flowcytometry. The injected and resident DC were identified as $\mathrm{CFSE}^{+} \mathrm{CD} 11 \mathrm{c}^{+}$or $\mathrm{CFSE}-\mathrm{CD} 11 \mathrm{c}^{+}$cells, respectively. Although, there were no significant difference between CFSE- CD11 $\mathrm{c}^{+}$cells homed to the draining inguinal $\mathrm{LNs}, \mathrm{CFSE}^{+} \mathrm{CD} 11 \mathrm{c}^{+} \mathrm{DC}$ homed to the draining inguinal LNs in radiation group is much less than control group (Figure 7), which indicates that radiation inhibit DC migration in vivo. This data indicate that $\gamma$-ray exposure could inhibit DC migration not only in vitro, but also in vivo. 


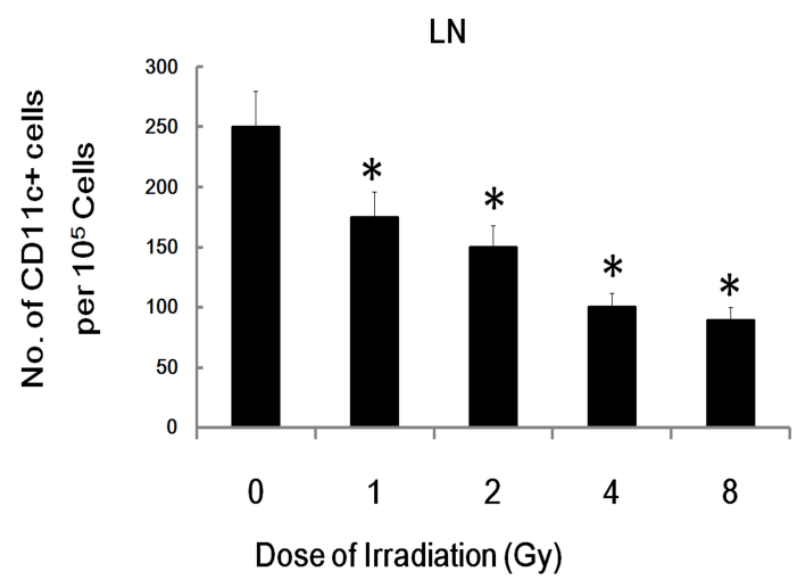

Figure $7 \gamma$-ray exposure inhibits DC migration in vivo. Day 5 DCs were labeled with CFSE and then treated with 0 , I, 2, 4 or $8 \mathrm{~Gy}$ of IR. Then $10^{6}$ CFSE-labeled DC were injected into C57BL/6 mice. 24 hours later, the injected site of skin and the draining inguinal LN (lymph node) were harvested and processed as described previously [ I7, 24, 25]. Single cell suspensions from the draining inguinal LN were prepared, stained with CD I Ic-PE (eBioscience) and analyzed by FACS described in the Materials and Methods. Figure showed statistical data of CDI Ic and CFSE double positive cell number from the draining inguinal LN of C57BL/6 mice injected with different treated DCs. Relative Data are shown as the means \pm standard deviation from triplicate experiments. *: $\mathrm{P}<0.05$.

\section{Discussion}

It is generally accepted that high doses of ionizing radiation can cause immunosuppression and it is also well known that the leading cause of acute radiation sickness is apoptosis of GI tract lining which causes nausea and diarrhea [22, 23, 29]. While, immunosuppression and potential infections are also important cause of IR induced death [30]. However, the influence of ionizing irradiation on the functions of DC, the most potent APC, is largely ignored. DC migration, one critical step for mature DC-initiated immune response, is orchestrated by a complex interplay between chemokines and their receptors [31]. During the differentiation and maturation of DC, sequential and orchestrated expressions of chemokine receptors are responsible for the proper migration of DC to immune sites [15]. Upon DC maturation, upregulation of CCR7 will direct immigration of DC into the lymph nodes or spleen. The chemokine receptor CCR7 and its ligands CCL19 and CCL21 control myeloid DC migration into different lymphatic vessels and the localization of these DC within LN $[17,20,21]$. Our data shows that $\gamma$ radiation exposure can inhibit LPS-induced upregulation of CCR7 expression in DC, and accordingly, gamma ray-exposed DC exhibited impaired migration ability to CCL19/MIP-3 in vitro. Consistently, $\gamma$ irradiation could also down-regulated PGE2 secretion by DC. Our data also shows that exogenous PGE2 reduces the $\gamma$ radiation-induced mi- gratory impairment of DC and partially restores CCR7 expression. This is consistent with results by other groups that exogenous PGE2 can restore CCR7 expression and migratory capacity reported [20].

Interestingly, exogenous PGE2 can only partially reduce $\gamma$ radiation-induced migratory impairment and only partly restore CCR7 expression in DC. Furthermore, we also found that PGE2 restores CCR7 expression much more effectively than rescues of migratory impairment of DC induced by IR; PGE2 also acts more effectively on DC irradiated at 2 Gy than 8 Gy. These data indicate that the PGE2-CCR7 pathway is not the only mechanism of IR induced DC migration impairment; this is different with other agents that inhibit DC migration which only impairs the CCR7 expression. We also found that IR can induce DC apoptosis and inhibit DC viability in a dose dependent manner. Although this part maybe not a [32] totally new finding, our data also suggests that $\gamma$ radiation induced apoptosis also participates in DC migration impairment.

This mechanism of inhibitory effect by IR on DC migration, due to DC apoptosis and down-regulation of CCR7 expression, is important because we can't just protect DC from cell death or CCR7 down-regulation on $\gamma$ ray-medicated DC migration defects, we must take into account the two pathways.

Our data indicate that $\gamma$-ray exposure could inhibit DC migration not only in vitro, but also in vivo. This is very important because the importance of DC 
in immune regulation. Studies also show that one of the major immunological concerns about radiation induced cell death is the increased production of transforming growth factor-b (TGF- $\beta$ ). In fact, Ionizing radiation has been shown to release TGF- $\beta$ in vitro and in vivo [32]. In normal tissues TGF- $\beta$ leads to growth inhibition, apoptosis, and serve as a tumor suppressor gene [33]. However, TGF- $\beta$ expression is often increased in many tumors and it can play some oncogene like function [34]. In our system, PGE2 and IL10 production by DC were also been found to be significantly inhibited by $\gamma$ ray; However, we didn't found $\gamma$ ray can significantly inhibit or enhance LPS-induced TGF- $\beta$ production by DCs by using ELISA assay (data not shown). Maybe our system is a little different from previous studies and we will explore this issue later.

In our current study, IL-10 has been found to be reduced by irradiation. It is true that IL-10 is well known for immunosuppression function [1, 11]. However, studies also show that IL-10 may play important role in killing acute influenza virus [35]. Therefore, our data indicates that IL-10 may not play important role in radiation induced migration impairment of DCs. However, it will play immunosuppresing roles in other process.

In summary, our results demonstrate that $\gamma$ radiation potently inhibits LPS-triggered CCR7 expression and induces apoptosis of DC, leading to the impaired DC migration to CCL19 in vitro and in vivo. The DC migration impaired by gamma ray exposure may be partly prevented by exogenous PGE2. These results provide an important mechanistic insight into $\gamma$ radiation medicated immunosuppression.

\section{Acknowledgment}

We thank Dr. Cindy Savage \& Dr. Trip Barthel for critically reading of the manuscript. Special thanks to Dr. Xuetao Cao from National Key Laboratory of Medical Immunology and Dr. Pu You from department of cell biology of our university for providing additional helps. We wish to thank Dr. Min Li from the department of hygienics of our university for doing the q-RT-PCR assays.

Funding: This work was supported in part by the grants from National Natural Science Foundation of China (No. 30970679; No. 31070762 \& No. 81001221). The funders had no role in study design, data collection and analysis, decision to publish, or preparation of the manuscript.

\section{Conflict of Interests}

The authors have declared that no conflict of interest exists.

\section{References}

1. Banchereau J and Steinman R.M. Dendritic cells and the control of immunity. Nature, 1998. 392(6673): 245-52.

2. Zitvogel L. Dendritic and natural killer cells cooperate in the control/switch of innate immunity. J Exp Med, 2002. 195(3): F9-14.

3. Banchereau J, et al. Immunobiology of dendritic cells. Annu Rev Immunol, 2000. 18: 767-811.

4. Lanzavecchia A and Sallusto F. Regulation of T cell immunity by dendritic cells. Cell, 2001. 106(3): 263-6.

5. Garg $S$, et al. Genetic tagging shows increased frequency and longevity of antigen-presenting, skin-derived dendritic cells in vivo. Nat Immunol, 2003. 4(9): 907-12.

6. Mellman I and Steinman R.M. Dendritic cells: specialized and regulated antigen processing machines. Cell, 2001. 106(3): 255-8.

7. Zhang $Z$, et al. Differential restoration of myeloid and plasmacytoid dendritic cells in HIV-1-infected children after treatment with highly active antiretroviral therapy. Immunol J, 2006. 176(9): 5644-51.

8. Allan R.S, et al. Epidermal viral immunity induced by CD8alpha+ dendritic cells but not by Langerhans cells. Science, 2003. 301(5641): 1925-8.

9. Shlomchik W.D, et al. Prevention of graft versus host disease by inactivation of host antigen-presenting cells. Science, 1999. 285(5426): 412-5.

10. Tacken P.J, et al. Dendritic-cell immunotherapy: from ex vivo loading to in vivo targeting. Nat Rev Immunol, 2007. 7(10): 790-802.

11. Hackstein $\mathrm{H}$ and Thomson A.W. Dendritic cells: emerging pharmacological targets of immunosuppressive drugs. Nat Rev Immunol, 2004. 4(1): 24-34.

12. Merad $\mathrm{M}$, et al. Langerhans cells renew in the skin throughout life under steady-state conditions. Nat Immunol, 2002. 3(12): $1135-41$

13. Naik S.H, et al. Development of plasmacytoid and conventional dendritic cell subtypes from single precursor cells derived in vitro and in vivo. Nat Immunol, 2007. 8(11): 1217-26.

14. Onai $\mathrm{N}$, et al. Identification of clonogenic common Flt3+M-CSFR+ plasmacytoid and conventional dendritic cell progenitors in mouse bone marrow. Nat Immunol, 2007. 8(11): 1207-16.

15. Faure-Andre G, et al. Regulation of dendritic cell migration by CD74, the MHC class II-associated invariant chain. Science, 2008. 322(5908): 1705-10.

16. Randolph G.J. Dendritic cell migration to lymph nodes: cytokines, chemokines, and lipid mediators. Semin Immunol, 2001. 13(5): 267-74.

17. Ohl L, et al. CCR7 governs skin dendritic cell migration under inflammatory and steady-state conditions. Immunity, 2004. 21(2): 279-88.

18. Forster R, et al. CCR7 coordinates the primary immune response by establishing functional microenvironments in secondary lymphoid organs. Cell, 1999. 99(1): 23-33.

19. Kabashima K, et al. Prostaglandin E2-EP4 signaling initiates skin immune responses by promoting migration and maturation of Langerhans cells. Nat Med, 2003. 9(6): 744-9.

20. Scandella E, et al. Prostaglandin E2 is a key factor for CCR7 surface expression and migration of monocyte-derived dendritic cells. Blood, 2002. 100(4): 1354-61. 
21. Scandella E, et al. CCL19/CCL21-triggered signal transduction and migration of dendritic cells requires prostaglandin E2. Blood, 2004. 103(5): 1595-601.

22. Vrijheid M, et al. Ionizing radiation and risk of chronic lymphocytic leukemia in the 15-country study of nuclear industry workers. Radiat Res, 2008. 170(5): 661-5.

23. Burdelya L.G, et al. An agonist of toll-like receptor 5 has radioprotective activity in mouse and primate models. Science, 2008. 320(5873): 226-30.

24. Zhang M, et al. Splenic stroma drives mature dendritic cells to differentiate into regulatory dendritic cells. Nat Immunol, 2004. 5(11): 1124-33.

25. Chen $\mathrm{T}$, et al. Cyclosporin A impairs dendritic cell migration by regulating chemokine receptor expression and inhibiting cyclooxygenase-2 expression. Blood, 2004. 103(2): 413-21.

26. Fu Z, et al. Expression changes of ERK1/2, STAT3 and SHP-2 in bone marrow cells from gamma-ray induced leukemia mice. J Radiat Res (Tokyo), 2006. 47(2): 121-30.

27. Qian L, et al. Radioprotective effect of hydrogen in cultured cells and mice. Free Radic Res, 2010. 44(3): 275-82.

28. Qian $\mathrm{L}$, et al. The potential cardioprotective effects of hydrogen in irradiated mice. J Radiat Res (Tokyo), 2010. 51(6): 741-7.

29. Ward J.F. DNA damage produced by ionizing radiation in mammalian cells: identities, mechanisms of formation, and reparability. Prog Nucleic Acid Res Mol Biol, 1988. 35: 95-125.

30. Kalpana K.B, et al. Investigation of the radioprotective efficacy of hesperidin against gamma-radiation induced cellular damage in cultured human peripheral blood lymphocytes. Mutat Res, 2009. 676(1-2): 54-61.

31. Merad M, Ginhoux F., and Collin M. Origin, homeostasis and function of Langerhans cells and other langerin-expressing dendritic cells. Nat Rev Immunol, 2008. 8(12): 935-47.

32. Barcellos-Hoff M.H, et al. Transforming growth factor-beta activation in irradiated murine mammary gland. J Clin Invest, 1994. 93(2): 892-9.

33. Derynck R, Akhurst R.J., and Balmain A. TGF-beta signaling in tumor suppression and cancer progression. Nat Genet, 2001. 29(2): 117-29.

34. Bachman K.E and Park B.H. Duel nature of TGF-beta signaling: tumor suppressor vs. tumor promoter. Curr Opin Oncol, 2005. 17(1): 49-54.

35. Sun J, et al. Effector $T$ cells control lung inflammation during acute influenza virus infection by producing IL-10. Nat Med, 2009. 15(3): 277-84. 